\title{
Exploring Hidden Issues in the Use of Antipsychotic Polypharmacy in the Treatment of Schizophrenia
}

\author{
Jung-Jin Kim', Chi-Un Pae ${ }^{1,2}$, Changsu Han ${ }^{3}$, Won-Myong Bahk', Soo-Jung Lee', \\ Ashwin A. Patkar ${ }^{4}$, Prakash S. Masand ${ }^{5}$ \\ ${ }^{1}$ Department of Psychiatry, ${ }^{2}$ Cell Death Disease Research Center, College of Medicine, The Catholic University of Korea, ${ }^{3}$ Department of \\ Psychiatry, Korea University College of Medicine, Seoul, Korea, ${ }^{4}$ Department of Psychiatry and Behavioral Sciences, Rush University Medical \\ Center, Chicago, IL, USA, ${ }^{5}$ Global Medical Education, New York, NY, USA
}

\begin{abstract}
The mainstay of schizophrenia treatment is pharmacological therapy using various antipsychotics including first- and second-generation antipsychotics which have different pharmacokinetic and pharmacodynamic property leading to differential presentation of adverse events (AEs) and treatment effects such as negative symptoms, cognitive symptoms and cormorbid symptoms. Major treatment guidelines suggest the use of antipsychotic monotherapy (APM) as a gold standard in the treatment of schizophrenia. However, the effects of APM is inadequate and less potent to achieve symptom remission as well as functional recovery in real practice which has been consistently reported in numerous controlled clinical trials, large practical trials, independent small studies and systematic reviews till today. Therefore antipsychotic polypharmacy (APP) regardless of the class of antipsychotics has been also commonly utilized for many reasons in real world practice. However, APP has also crucial pitfalls including increase of total psychotics including antipsychotics, high-doses of antipsychotics used, poor compliance, drug-drug interaction and risks for developing AEs, all of which are paradoxically related to poor clinical outcomes, whereas APP has also substantial advantages in reduction of re-hospitalization, severe psychopathology and targeted control of concurrent symptoms. Given currently limited therapeutic options, it is also important to properly utilize APP in order to maximize its clinical utility and minimize its risk for better treatment outcomes for patients with schizophrenia, based on risk/benefit with full understanding of pharmacological and clinical issues on APP. The present paper intends to address intriguing and important issues in the use of APP in real world practice.
\end{abstract}

KEY WORDS: Antipsychotics; Schizophrenia; Polypharmacy; Issue.

\section{INTRODUCTION}

Schizophrenia is a chronic disabling mental illness requiring enormous endeavor of medical professionals, family and public supports and costs since it is not easily cured and needs a long-term care due to its fluctuating and deteriorating clinical course over time [1]. The mainstay of the treatment for schizophrenia has been needless

Received: December 2, 2020 / Revised: January 13, 2021

Accepted: February 2, 2021

Address for correspondence: Chi-Un Pae

Department of Psychiatry, Bucheon St. Mary's Hospital, College

of Medicine, The Catholic University of Korea, 327 Sosa-ro,

Wonmi-gu, Bucheon 14647, Korea

E-mail: pae@catholic.ac.kr

ORCID: https://orcid.org/0000-0003-1632-4248 to say pharmacological agents including antipsychotics. Indeed, the development and introduction of second-generation antipsychotics possessing differential pharmacodynamic (PD) property in comparison with the earlier, first-generation antipsychotics have significantly improved the biological treatment options in routine practice, providing better tolerability and safety which are substantially and critically involved in medication compliance and persistence [2].

However, the efficacy of antipsychotic therapy is not satisfactory in real world by which many next treatment strategies have been utilized in routine practice for offering better treatment outcomes for patients with schizophrenia, although some second treatment options such as antipsychotic polypharmacy (APP) are not officially rec-

@) This is an Open-Access article distributed under the terms of the Creative Commons Attribution Non-Commercial License (http://creativecommons.org/licenses/by-nc/4.0) which permits unrestricted non-commercial use, distribution, and reproduction in any medium, provided the original work is properly cited. 
ommended by major treatment guidelines or fully supported by evidence-based data from randomized controlled, clinical trials (RCTs), cohort study and adequately-powered, well-designed practical trials [3-10].

Despite antipsychotic monotherapy (APM) is the goldstandard therapy recommended by well-known treatment guidelines from many popular and influential academic societies, APP has been also widely used for some proportion of patients with schizophrenia, for instance APP is commonly utilized and considered for treating difficultto-treat cases or intolerance/failure to initial APM in naturalistic treatment settings $[8,11-13]$. In fact, the prevalence of APP showed quite wide ranges due to differences in methodological approach and samples included in such studies. The global median rate of APP was reported approximately $20 \%$ in a recent systematic review involving 147 studies [14], although there have been substantial variations among geographic regions [2,15-17].

Why do clinicians utilize APP for treatment of their patients with schizophrenia? Many persuasive reasons were reported in numerous studies where further control of psychotic symptoms especially positive symptoms ranked as the most important reason. Enhancement of cognitive impairment, decrease of readmission, prevention of relapse/recurrence, avoidance of high-dose AP therapy, overlap for AP switch, target treatment for specific and/or comorbid symptoms including anxiety, sleep disturbance, depression, violence, and agitation, counteract adverse events (AEs) using differential PD profile [18-22]. However, APP has also clear disadvantages including diverse AEs such sedation, increased rate of metabolic syndrome, somnolence, reduced concentration and extrapyramidal symptoms (EPSs), complexity of treatment regimen, drug-drug interaction, high medical costs, unclear determination on treatment response among APs, poor compliance/persistence to treatment, and increased total doses of APs [20,23-25].

Apart from debate on whether APP is necessary or excessive treatment approach beyond major treatment guidelines recommended by academic or regulatory authorities and/or it is adequately supported by sufficient evidence from large RCTs, APP has already positioned as one of routine practices. If $\mathrm{so}$, it should be wise and realistic to discuss about hidden points in the use of APP for real world practice. Thus, the present paper elucidates some intriguing and practical points considered in the uti- lization of APP in routine practice.

\section{WHEN AND HOW TO INITIATE APP?}

Most treatment guidelines state that APM should be the first-line treatment and APP should be a treatment strategy of last resort for treatment-resistant schizophrenia [2-10]. They stated that clozapine should be tried in the first place to overcome inadequate treatment response from different APM trials. At least two or more APM failures lasting for more than 4-month for each APM are usually required for proceeding to next treatment options [9].

Addition of another AP to clozapine should be an easy next step when patients have already shown a partial response. Indeed, many studies have shown beneficial effects of APP combing clozapine and another AP. According to a meta-analysis [26] investigating 4 RCTs, addition of another AP to preexisting clozapine was found to be beneficial to cases with adequately-tested prior APM for at least 4-6 weeks, indicating a premise (adequate duration of prior APM) should be mandatory to achieve optimal clinical outcomes.

Such findings were also replicated in the subsequent meta-analysis $[27,28]$ and independent studies $[29,30]$. APP can be started in specific clinical situations in reality as follows: a failure to at least two or more APM, a failure to clozapine, partial response to clozapine, intolerance to APM including clozapine, clozapine-contraindicated case, counteract AEs from initial or another AP in APP regimen.

As for doses of individual AP in the use of APP, there has been no well-evidenced data to support whether clinicians should escalate the dose of later AP up to maximal therapeutic dose to achieve treatment response as targeted in combination with initial existing AP. When reflecting one of the advantages of APP is to prevent excessive use of high-dose APM leading to serious AEs or poor compliance, minimal but effective dose of later AP (compatible with $\mathrm{D}_{2}$ occupancy of $65 \%$ ) should be optimal along with regular assessment using measurementbased evaluation on patients' psychotic symptoms, which will definitely protect patients from unnecessary sufferings which can be potentially caused by overdoses of APs. 


\section{TOTAL NUMBER OF APP OR TOTAL DAILY DOSE OF APP, WHICH IS MORE PROBLEMATIC?}

APP results in an increased number of daily pills leading to high-dose AP therapy in routine practice. The total AP dose of APP is especially problematic when combining APs with high $D_{2}$ receptor affinity and blocking effects for treating patients since it will cause cumulative impacts on the appearance of unwanted side effects (SEs) such as EPS [12]. Significant relationship has been consistently between antipsychotic effect (including SEs) and degree of $\mathrm{D}_{2}$ receptor occupancy [31].

In fact, patients with EPS had significantly higher $\mathrm{D}_{2}$-receptor occupancy than those without, indicating the harmful effects of total dose on the development of EPS. Overall APs are known to show balanced efficacy with $\mathrm{D}_{2}$ occupancy of $65 \%$, while the development of SEs such as hyperprolactinemia and EPS may increase when $\mathrm{D}_{2}$ receptor occupancy exceeds $72 \%$, and $78 \%$, respectively, although inter-individual differences exist in $\mathrm{D}_{2}$ receptor occupancy [31,32].

In addition, $\mathrm{D}_{2}$ occupancy levels were significantly related with the vigilance and the summary scores in the measure of neurocognitive functions in the largest practical clinical trial, the Clinical Antipsychotic Trials in Intervention Effectiveness (CATIE) trial [33]. The results clearly demonstrated a nonlinear relationship between prescribed AP doses and overall neurocognitive function and vigilance; they were especially impaired in subjects who showed $D_{2}$ receptor occupancy level approximately, of $>80 \%$. Therefore, we may confirm that $\mathrm{D}_{2}$ occupancy of $>80 \%$ not only increases the risk for EPSs as consistently reported in the literature but also increases the risk for cognitive impairment [33]. These are in line with the results from other APP studies that demonstrated the total dose effects of AP in association with cognitive dysfunctions between APP and APM [34,35], favoring APM over APP.

Likewise, it was also found that AP total dose also mediates the association between polypharmacy and QTC interval [36]. In the study, the Baron and Kenny mediational approach with adjustment for confounding variables demonstrated that APP was significantly associated with QTc prolongation, APP was also significantly related with AP dose, AP dose was also significantly associated with
QTc prolongation, however, APP was no longer associated with QTc prolongation under control of AP dose, which clearly indicate the crucial determinant of QTC prolongation was the AP dose but not number of APs in APP [36].

An interesting recent Swedish cohort study with the first-episode schizophrenia found that excess and overall cardiovascular mortality in schizophrenia could be influenced by other underlying biological and clinical factors rather than $\mathrm{AP}$ treatment when used in adequate dosages [37]. According to the results, small to moderate AP dosage uses were associated with substantially lower mortality than high dose AP usage where a high dose AP usage was in particular significantly associated with a higher mortality in female than in male patients. The subsequent study also clearly replicated the critical impact of inappropriate use with high dose of APs on overall mortality in schizophrenia patients [38].

\section{IS INSIGHT ONE OF ISSUES IN THE USE OF APP?}

Poorer insight of schizophrenia patients was found to be significantly associated with more likelihood of receiving APP in admission in a Italian large study $(n=1,022)$ [39], while patients with APM presented good insight in the study. Despite such finding presenting a direct association between insight and APP, it should be weighed since insight is critically involved in many treatment issues in schizophrenia patients. Indeed, insight is one of crucial components determining medication persistence and adherence of schizophrenia patients, which is very important in the treatment of schizophrenia since AP maintenance therapy is the gold standard and principle in the treatment for schizophrenia and the negative relationship between insight and treatment adherence is consistently reported in schizophrenia patients $[40,41]$.

The strong link between impaired insight and nonadherence to AP was also replicated in the CATIE trial [42]. In the study, patients were classified by three groups depending on their insight such as no impairment (Positive and Negative Syndrome Scale [PANSS] G12=1), minimal impairment (PANSS G12 $=2-3$ ), and moderate-to-severe insight impairment (PANSS G12 $\geq 4$ ). According to the results, the adherence rates were $17 \%, 20 \%$, and $25 \%$ of patients with no, minimal, and moderate-to-severe insight 
impairment at month-6 after study initiation, and 31\%, $37 \%$, and $43 \%$ at month- 18 . The time to nonadherence was also significantly shorter in patients with moderate-to-severe insight impairment (13.5 months) than in patients with minimal (14.4 months) or no impairment (15.1 months) [42]. A prior longitudinal analysis of the CATIE data also showed a strong relationship between insight and development of positive attitudes toward AP treatment which is directly associated with substantial improvement of clinical outcomes including psychopathology, community functioning and greater medication compliance in schizophrenia patients [43]. Therefore, we reasonably assume the strong relationship between insight, adherence and APP in the treatment of schizophrenia.

\section{IS THERE ANY BEST-AVAILABLE APP REGIMEN FOR TREATMENT OF SCHIZOPHRENIA?}

There has been scarce data regarding whether one specific APP regimen should have a superiority over another APP regimen. Interestingly the longest and largest cohort study ( $n=62,250$ ) followed 20-year [24] has found that significantly lower psychiatric rehospitalization among all 29 different APM and APP types was found in clozapine plus aripiprazole APP, resulting in $14 \%$ to $23 \%$ lower risk of rehospitalization than clozapine APM. Furthermore such difference in psychiatric rehospitalization between clozapine plus aripiprazole APP and clozapine APM was more profound in first-episode patients favoring clozapine plus aripiprazole APP over clozapine APM (hazard ration $=0.78$ and $22 \%$ difference). Any APP presented a $7 \%$ to $13 \%$ lower risk of psychiatric rehospitalization compared with any APM, indicating that rational APP excluding clozapine plus aripiprazole could be also clinically useful treatment strategy regardless of combined APs as APP regimen, especially with the use of two different APs possessing different types of receptor profile (i.e., $\mathrm{D}_{2}$ receptor antagonist + partial agonist; $\mathrm{D}_{2}$ receptor tight binding agent + loose $\mathrm{D}_{2}$ receptor binding agent).

\section{HOW LONG SHOULD WE MAINTAIN APP IN ROUTINE PRACTICE?}

A previous study evaluated the prescription trend of AP switch and APP use in the real-treatment settings from a longitudinal perspective [44]. In the study, APM was started with 208 patients in which $34.1 \%$ of the patients left APM and switched to different APs (27.4\%) and/or APM $(17.8 \%)$ within 2 years. Intriguingly inadequate efficacy of AP was the main reason for AP switch, however, it was improperly conducted since the APM dose was below the recommended range in $47.4 \%$ of the AP switch and APP was also prematurely used after a median of one AP trial for only 3 months even in AP-naïve patients, indicating that clinicians might utilize AP switch and APP not based on treatment guidelines or consensus but possibly by empirical, preferential, and easy approach as a next treatment step.

In fact, there have been a dearth of data regarding how long APP can last for treatment of schizophrenia. Symptomatic remission has different criteria using psychometric assessment such as PANSS, the Scale for the assessment of negative symptoms and positive symptoms (SANS/SAPS), and the Brief Psychiatric Rating Scale (BPRS) [45]. It can be evaluated with 8 items in the PANSS (delusions, unusual thought content, hallucinatory behavior, conceptual disorganization, mannerisms/posturing, blunted affect, passive/apathetic social withdrawal, and lack of spontaneity and flow of conversation), all of which have to be simultaneously scored with mild or less symptom severity ( $\leq 3$ points). Use of BPRS (severity $\leq 3$ points) criteria may be supplemented by use of the SANS (severity $\leq$ 2) points criteria for evaluating overall remission since it does not include assessment of negative symptoms $[45,46]$. The cut-off time for evaluation whether patients remitted from psychotic symptoms of schizophrenia are usually 6-month [45] by which we can assume that APP transition to APM should be ready or prepared around 6-month upon achieving symptomatic remission in a patient with schizophrenia; however, it should be properly determined based on a patient's current clinical situation since symptomatic remission can be achieved and maintained for a certain period and but functional recovery may not be sufficient yet. Indeed, many working groups [47-51] tested such time differences between symptomatic remission and functional recovery in patients with schizophrenia.

Indeed, a significant difference in functional capacity between remitters and nonremitters does not necessarily mean that remitters are functioning well. In addition, vocational/occupational status is possibly influenced by others 
factors not depending on remission status [46]. Furthermore, it has been known that first- and multiple-episode schizophrenia may have differential chances of fulfillment of remission. According to Dr. Lambert and colleagues' [46] excellent review that analyzed more than 30 studies, they concluded that first-episode patients display higher remission frequencies during follow-up ( $48 \%$ vs. $43 \%$ ) when comparing first- and multiple episode completers, using the severity and time remission criteria and there is an increase of remission frequencies between 6-month and 24-month follow-ups (6-month: 46\%, 12-month: 52\%, 24-month: 63\%) when using the severity remission criteria only in both groups. These findings clearly point out that remission frequency could be different by episode of schizophrenia favoring first-episode over multiple episodes and also much longer time should be also considered as a cut-off time for the determination/ assessment of remission status in practice.

In a small retrospective study, it was found that APP ranged from 6 months to surprisingly, greater than 9 years [52]. In a recent mental healthcare database study, the long-term APP for more 6-month use was $23.1 \%$ among 10,945 cases with 4.5 years' mean follow-up [53].

Therefore, it should be very difficult for clinicians to establish exact time for APP (using two APs) transition to APM or for discontinuation of $\mathrm{AP}(\mathrm{s})$ among multiple APs-used APP (more than 3 APs) based on currently available, limited evidence and numerous clinical factors involved with APP. Such duration issue of APP been also confronted in several APP trials using clozapine and another AP $[26,27]$. Hence it should be reasonable to say that when a patient remits from a full domain of psychotic symptoms and maintain clinical stability over 6 months, careful and gradual discontinuation of one AP among APP regimen (i.e., two APs) can be considered for switching to APM or for reduction of APP regimen (i.e., more than 3 APs) in accordance with clinical status of individual case. On the other hand, long-standing maintenance of APP can be also justifiable when relapse occurs upon switching to APM [8]. When reflecting a need of personalized treatment and highly diverse clinical course of schizophrenia case-by-case, it should be more pertinent that clinicians should ascertain the lowest effective AP dosages in the use of APP to optimally prevent both relapses and $\mathrm{AEs}$, and to allow optimal functional recovery, rather than being troubled by determining wheth- er to maintain or discontinue antipsychotics $[13,54]$.

\section{HOW TO SWITCH APP TO APM IN CLINICAL PRACTICE?}

In some studies, APP stay or switch to APM were tested. According to the previous systematic review including one RCT and two open-label trials [55], in two of three studies, more than two thirds of the subjects successfully moved from APP to APM (40/58, 69.0\%; 34/44, and $77.3 \%$, respectively) indicating that switch from APP to APM may appear feasible in a majority of schizophrenia patients in routine practice. Essock et al. [56] has also tested the feasibility of switching to APM from APP in a 6-month randomized and naturalistic follow-up study where all-cause discontinuation rate and time were more frequent and shorter in switch to APM than in APP stay, however, two-thirds of patients succeeded in switch to APM without group difference in worsening of psychotic symptoms. To address knowledge gap on APP stay and switch to APM, another 12-week RCT compared two treatment strategies of APP stay and switch to APM [57]. Seventeen and eighteen patients who have been on APP with stabilized doses for each AP were randomly assigned to either APP stay (continuation of two APs) or switch to APM (discontinuation of later AP), respectively. The primary endpoint was the change of BPRS score from baseline to the end of treatment, where early drop-out related with clinical worsening occurred in $5.8 \%$ of patients with APP stay, while $22.2 \%$ of patients with switch to APM. However, there was no indication of clinical worsening with switch to APM as measured by BPRS and Clinical Global Impression-Severity scores. Approximately $80 \%$ $(n=14)$ of patients with APP was able to be safely switched to APM with no clinical deterioration, in which the risk for clinical worsening was prominent in the first few several months.

However, such successful switch to APM from APP was challenged in a 7 multi-center study by Constantine et al. [58]. One hundred and four $(n=104)$ stable outpatient schizophrenia on 2 APs were randomly assigned to stay on APP or to switch to APM, where patients switched to APM showed greater symptom worsening than patients with APP stay. Such differences were prominent in the second 6months of the study. All-cause discontinuation rates over the 1-year trial were also significantly higher in 
the switch to APM group than in the APP stay group (42\% vs. $13 \%)$.

More interestingly the risks and benefits of switching from APP with two non-clozapine oral APs and APP involving either clozapine or an injectable antipsychotic were investigated in a recent study [59]. In total, 90 psychotic patients were randomized to APP stay or to switch to APM. The assessment was performed every 2 months for one-year follow-up period. Switching group involving either clozapine or an injectable AP did not differ over time from APP stay group on either symptom control or SEs, while significantly more worsening of psychotic symptoms was found in switching group with two nonclozapine oral APs. This data suggests that APP involving clozapine or injectable APs may be easier and safer to be switched to APM rather than APP with two non-clozapine APs, possibly indicating differential risk of APP switch to APM depending on the regimen utilized.

In addition, according to the recent meta-analysis to find the predictors of successful AP dose reduction in schizophrenia including 37 trials [60], young age $(\leq 40$ years), short duration of illness ( $\leq 10$ years), and low post-reduction chlorpromazine equivalent dose (CPZeq) ( $\leq 200 \mathrm{mg} / \mathrm{d}$ ) were found to be significantly associated with risk of relapse. The relapse rate was significantly higher (risk ratio $[R R]=1.96$ ) in the AP dose reduction group than the AP dose maintenance group, whereas neuro-cognition significantly more improved in AP dose reduction group than in AP dose maintenance group. Clinical deterioration was mostly re-stabilized by increasing the dose to the baseline level in $87.5 \%$ of the studies. Interestingly a subgroup analysis clearly indicated that only a post-reduction CPZeq dose $\leq 200 \mathrm{mg} / \mathrm{d}$ was associated with an increased risk of relapse by 2.79 of RR. This data clearly indicates the AP dose reduction should be possible but clinicians need to keep the dose in therapeutic range of individual $\mathrm{AP}$, at least corresponding to CPZeq dose of $200 \mathrm{mg} / \mathrm{d}$ or more.

\section{DISCUSSION}

According to a survey study [61], clinicians preferring APP had more clinical experience, see more patients per week, and had their own specific APP regimen as well as being attending physicians rather than resident doctor, continuing APP of prior physicians (75\% was inherent from previous prescribers) and having little concerns on APP treatment strategy. However, there were no differences in justification and concerns in the use of APP. These data clearly suggest that most clinicians use APP under similar purpose for enhancement of treatment outcomes and with careful considerations for minimization of AEs regardless they prefer APP or not. Interestingly another survey has found that clinicians preferring APP shares similar concerns such as unexpected potential AEs of APP regardless they are heavy or light APP users $(<$ $30 \%$ of patients) [62]. Indeed, the AEs of AP use have not been intensively and widely studied in routine practice, however, a recent systematic review [63] including 53 researches has shown critical points regarding AEs in the use of APPs, stating that APP with longer duration may be significantly associated with increased frequency and greater severity of AEs, although it should be more replicated in subsequent long-term comparative studies, especially with high-dose APM.

Different perspectives have already started to recognize APP as one of proper and feasible next treatment options in the treatment of schizophrenia in routine practice. Some experts proposed active inclusion of APP with pragmatic recommendations into practice guidelines for schizophrenia [8,64]. Obviously, data concerning superiority of APP over APM or other treatment options for treatment of schizophrenia is still scarce today, no high-quality RCTs have proven such crucial issues, however, some subgroup of schizophrenia definitely benefits from APP rather than APM.

Meanwhile many proportions of APP patients can be also safely switched to APM or just stay in APP retaining similar efficacy after stabilization, and some proportion of APP patients also continue same treatment approach in a longstanding period without tolerability issues, indicating a potential utility of APP as long-term maintenance treatment option for schizophrenia.

The largest and longest nationwide cohort study has clearly revealed that no single APs or APPs excluding clozapine plus aripiprazole combination should supersede the efficacy of clozapine alone in terms of psychiatric hospitalization, which clearly pointed out the beneficial effects of specific APP on predefined or confined treatment targets. The relatively weak $\mathrm{D}_{2}$ antagonist properties of clozapine might be enhanced by aripiprazole, which provides greater affinity for $D_{2}$ receptors. This should be in 
Table 1. Consideration points in the use of antipsychotic polypharmacy in routine practice

APP is common and effective treatment option in clinical practice regardless of diagnosis

Thorough review for past and family AP response before instating APP

Active engagement of patients in decision of APP application

Regular assessment for clinical status would be useful for initiating APP or returning to APM

Clozapine and aripiprazole appear the most beneficial APP combination based on clinical trial data

Closely monitor the total dose of APP to avoid unnecessary and serious adverse events or adverse reactions

Consider long-acting injectable antipsychotics when clinical status is stabilized and upon transition to APM

A failure to at least two or more APM, a failure to clozapine, partial response to clozapine, intolerance to APM including clozapine,

clozapine-contraindicated case, counteract AEs

Consider reinstating immediate prior APP regimen if symptom worsening emerges when reducing APP regimen

Keep the total dose of APs up to chlorpromazine equivalent dose of $200 \mathrm{mg} / \mathrm{d}$ regardless of classes of APs when tapering off APP regimen

Gradual tapering of AP should be always kept in mind upon transition to APM or reduction of APP regimen

When reflecting a need of personalized treatment and diverse clinical course of schizophrenia, clinicians should decide the lowest effective AP

dosages in the use of APP

Strong relationship is found between insight, adherence and APP in the treatment of schizophrenia

APP, antipsychotic polypharmacy; AP, antipsychotic; APM, antipsychotic monotherapy; AEs, adverse events.

line with the fact that beneficial effects of APP may come from synergistic effects on differential PD profiles, although additional effects of different PK profile among APs combined might be also partly involved in such effects. Table 1 addresses some clinical points in the use of antipsychotic polypharmacy in routine practice [65-68].

Given mentioned, APP has never been far from our routine practice but it has been staying indeed popular for a long time in naturalistic treatment settings. Reflecting insufficient and limited treatment options for schizophrenia, pertinent, reasonable and well-targeted usage of APP based on currently available evidence should be more practical and realistic in the treatment of schizophrenia in routine clinical practice.

\section{- Conflicts of Interest}

No potential conflict of interest relevant to this article was reported.

\section{Author Contributions}

Conceptualization: Chi-Un Pae, Jung-Jin Kim, Changsu Han. Supervision: Soo-Jung Lee, Won-Myong Bahk, Ashwin A. Patkar, Prakash S. Masand. Writing-original draft: Jung-Jin Kim, Chi-Un Pae. Writing - review \& editing: Changsu Han, Soo-Jung Lee, Won-Myong Bahk, Ashwin A. Patkar, Prakash S. Masand.

\section{ORCID}

Jung-Jin Kim

Chi-Un Pae https://orcid.org/0000-0003-3899-5579

https://orcid.org/0000-0003-1632-4248
Changsu Han https://orcid.org/0000-0002-4021-8907

Won-Myong Bahk https://orcid.org/0000-0002-0156-2510

Soo-Jung Lee https://orcid.org/0000-0002-1299-5266

Ashwin A. Patkar https://orcid.org/0000-0003-0484-1301

Prakash S. Masand https://orcid.org/0000-0003-1973-9449

\section{REFERENCES}

1. Psychosis and schizophrenia in adults: prevention and management [Internet]. London: National Collaborating Centre for Mental Health; 2014 Feb 12 lupdated 2014 Mar 1; cited at 2020 Dec 2]. Available from: https://www.nice.org.uk/guidance/cg178.

2. Lee JS, Yun JY, Kang SH, Lee SJ, Choi JH, Nam B, et al. Korean medication algorithm for schizophrenia 2019, second revision: treatment of psychotic symptoms. Clin Psychopharmacol Neurosci 2020;18:386-394.

3. Hasan A, Falkai P, Wobrock T, Lieberman J, Glenthoj B, Gattaz WF, et al. World Federation of Societies of Biological Psychiatry (WFSBP) Guidelines for Biological Treatment of Schizophrenia, part 1: update 2012 on the acute treatment of schizophrenia and the management of treatment resistance. World J Biol Psychiatry 2012;13:318-378.

4. Barnes TR, Drake R, Paton C, Cooper SJ, Deakin B, Ferrier IN, et al. Evidence-based guidelines for the pharmacological treatment of schizophrenia: updated recommendations from the British Association for Psychopharmacology. J Psychopharmacol 2020;34:3-78.

5. Galletly C, Castle D, Dark F, Humberstone V, Jablensky A, Killackey E, et al. Royal Australian and New Zealand College of Psychiatrists clinical practice guidelines for the management of schizophrenia and related disorders. Aust N Z J Psychiatry 2016;50:410-472.

6. Lehman AF, Lieberman JA, Dixon LB, McGlashan TH, Miller $\mathrm{AL}$, Perkins DO, et al. Practice guideline for the treatment of patients with schizophrenia, second edition. Am J Psychiatry 
2004; $161(2$ Suppl):1-56.

7. Remington G, Addington D, Honer W, Ismail Z, Raedler T, Teehan M. Guidelines for the pharmacotherapy of schizophrenia in adults. Can J Psychiatry 2017;62:604-616.

8. Stahl SM. Emerging guidelines for the use of antipsychotic polypharmacy. Rev Psiquiatr Salud Ment 2013;6:97-100.

9. Keepers GA, Fochtmann LJ, Anzia JM, Benjamin S, Lyness JM, Mojtabai R, et al. The American Psychiatric Association practice guideline for the treatment of patients with schizophrenia. Am J Psychiatry 2020;177:868-872.

10. Pae CU, Han C, Bahk WM, Lee SJ, Patkar AA, Masand PS. Consideration of long-acting injectable antipsychotics for polypharmacy regimen in the treatment of schizophrenia: put it on the table or not? Clin Psychopharmacol Neurosci 2021; 19:434-448.

11. Mojtabai R, Olfson M. National trends in psychotropic medication polypharmacy in office-based psychiatry. Arch Gen Psychiatry 2010;67:26-36.

12. Freudenreich O, Goff DC. Antipsychotic combination therapy in schizophrenia. A review of efficacy and risks of current combinations. Acta Psychiatr Scand 2002;106:323-330.

13. Lin SK. Antipsychotic polypharmacy: a dirty little secret or a fashion? Int I Neuropsychopharmacol 2020;23:125-131.

14. Gallego JA, Bonetti J, Zhang J, Kane JM, Correll CU. Prevalence and correlates of antipsychotic polypharmacy: a systematic review and meta-regression of global and regional trends from the 1970s to 2009. Schizophr Res 2012;138:18-28.

15. Park JH, Hong JS, Kim SM, Min KJ, Chung US, Han DH. Effects of amisulpride adjunctive therapy on working memory and brain metabolism in the frontal cortex of patients with schizophrenia: a preliminary positron emission tomography/computerized tomography investigation. Clin Psychopharmacol Neurosci 2019;17:250-260.

16. Oltra JAE. Improving therapeutic interventions of schizophrenia with advances in stem cell technology. Clin Psychopharmacol Neurosci 2020;18:352-361.

17. Dong M, Zeng LN, Zhang Q, Yang SY, Chen LY, Najoan E, et al. Prescription of antipsychotic and concomitant medications for adult Asian schizophrenia patients: findings of the 2016 Research on Asian Psychotropic Prescription Patterns (REAP) survey. Asian J Psychiatr 2019;45:74-80.

18. Hatta K, Hasegawa H, Imai A, Sudo Y, Morikawa F, Katayama $\mathrm{S}$, et al. Real-world effectiveness of antipsychotic monotherapy and polytherapy in 1543 patients with acute-phase schizophrenia. Asian J Psychiatr 2019;40:82-87.

19. Guinart D, Correll CU. Antipsychotic polypharmacy in schizophrenia: why not? J Clin Psychiatry 2020;81:19ac13118.

20. Sun F, Stock EM, Copeland LA, Zeber JE, Ahmedani BK, Morissette SB. Polypharmacy with antipsychotic drugs in patients with schizophrenia: trends in multiple health care systems. Am J Health Syst Pharm 2014;71:728-738.

21. Galling B, Roldán A, Hagi K, Rietschel L, Walyzada F, Zheng $\mathrm{W}$, et al. Antipsychotic augmentation vs. monotherapy in schizophrenia: systematic review, meta-analysis and metaregression analysis. World Psychiatry 2017;16:77-89.

22. Tiihonen J, Taipale H, Mehtälä J, Vattulainen P, Correll CU, Tanskanen A. Association of antipsychotic polypharmacy vs monotherapy with psychiatric rehospitalization among adults with schizophrenia. JAMA Psychiatry 2019;76:499-507.

23. Roh D, Chang JG, Kim CH, Cho HS, An SK, Jung YC. Antipsychotic polypharmacy and high-dose prescription in schizophrenia: a 5-year comparison. Aust NZJ Psychiatry 2014;48: 52-60.

24. Aly El-Gabry DM, Abdel Aziz K, Okasha T, Azzam H, Okasha A. Antipsychotic polypharmacy and its relation to metabolic syndrome in patients with schizophrenia: an Egyptian study. J Clin Psychopharmacol 2018;38:27-33.

25. Takeuchi H, Suzuki T, Remington G, Uchida H. Antipsychotic polypharmacy and corrected QT interval: a systematic review. Can J Psychiatry 2015;60:215-222.

26. Paton C, Whittington C, Barnes TR. Augmentation with a second antipsychotic in patients with schizophrenia who partially respond to clozapine: a meta-analysis. J Clin Psychopharmacol 2007;27:198-204.

27. Taylor DM, Smith L, Gee SH, Nielsen J. Augmentation of clozapine with a second antipsychotic- a meta-analysis. Acta Psychiatr Scand 2012;125:15-24.

28. Wang J, Omori IM, Fenton M, Soares B. Sulpiride augmentation for schizophrenia. Cochrane Database Syst Rev 2010;(1): CD008125.

29. Munro J, Matthiasson P, Osborne S, Travis M, Purcell S, Cobb AM, et al. Amisulpride augmentation of clozapine: an open non-randomized study in patients with schizophrenia partially responsive to clozapine. Acta Psychiatr Scand 2004;110: 292-298.

30. Assion HJ, Reinbold H, Lemanski S, Basilowski M, Juckel G. Amisulpride augmentation in patients with schizophrenia partially responsive or unresponsive to clozapine. A randomized, double-blind, placebo-controlled trial. Pharmacopsychiatry 2008:41:24-28.

31. Kapur S, Zipursky R, Jones C, Remington G, Houle S. Relationship between dopamine $D_{2}$ occupancy, clinical response, and side effects: a double-blind PET study of first-episode schizophrenia. Am J Psychiatry 2000;157:514-520.

32. Nordström AL, Farde L, Wiesel FA, Forslund K, Pauli S, Halldin C, et al. Central $D_{2}$-dopamine receptor occupancy in relation to antipsychotic drug effects: a double-blind PET study of schizophrenic patients. Biol Psychiatry 1993;33:227235.

33. Sakurai H, Bies RR, Stroup ST, Keefe RS, Rajji TK, Suzuki T, et al. Dopamine $D_{2}$ receptor occupancy and cognition in schizophrenia: analysis of the CATIE data. Schizophr Bull 2013;39: 564-574.

34. Elie D, Poirier M, Chianetta J, Durand M, Grégoire C, Grignon S. Cognitive effects of antipsychotic dosage and polypharmacy: a study with the BACS in patients with schizophrenia and 
schizoaffective disorder. J Psychopharmacol 2010;24:10371044.

35. Hori H, Yoshimura R, Katsuki A, Hayashi K, Ikenouchi-Sugita A, Umene-Nakano W, et al. Several prescription patterns of antipsychotic drugs influence cognitive functions in Japanese chronic schizophrenia patients. Int I Psychiatry Clin Pract 2012;16:138-142.

36. Barbui C, Bighelli I, Carrà G, Castellazzi M, Lucii C, Martinotti $\mathrm{G}$, et al. Antipsychotic dose mediates the association between polypharmacy and corrected QT interval. PLoS One 2016;11: e0148212.

37. Torniainen M, Mittendorfer-Rutz E, Tanskanen A, Björkenstam C, Suvisaari J, Alexanderson K, et al. Antipsychotic treatment and mortality in schizophrenia. Schizophr Bull 2015;41:656663.

38. Tiihonen J, Mittendorfer-Rutz E, Torniainen M, Alexanderson K, Tanskanen A. Mortality and cumulative exposure to antipsychotics, antidepressants, and benzodiazepines in patients with schizophrenia: an observational follow-up study. Am J Psychiatry 2016;173:600-606.

39. Santone G, Bellantuono C, Rucci P, Picardi A, Preti A, de Girolamo G. Patient characteristics and process factors associated with antipsychotic polypharmacy in a nationwide sample of psychiatric inpatients in Italy. Pharmacoepidemiol Drug Saf 2011;20:441-449.

40. Czobor P, Van Dorn RA, Citrome L, Kahn RS, Fleischhacker WW, Volavka J. Treatment adherence in schizophrenia: a patient-level meta-analysis of combined CATIE and EUFEST studies. Eur Neuropsychopharmacol 2015;25:1158-1166.

41. Misdrahi D, Tessier A, Swendsen J, Berna F, Brunel L, Capdevielle D, et al. Determination of adherence profiles in schizophrenia using self-reported adherence: results from the FACE-SZ dataset. J Clin Psychiatry 2016;77:e1130-e1136.

42. Kim J, Ozzoude M, Nakajima S, Shah P, Caravaggio F, Iwata $\mathrm{Y}$, et al. Insight and medication adherence in schizophrenia: an analysis of the CATIE trial. Neuropharmacology 2020;168: 107634.

43. Mohamed S, Rosenheck R, McEvoy J, Swartz M, Stroup S, Lieberman JA. Cross-sectional and longitudinal relationships between insight and attitudes toward medication and clinical outcomes in chronic schizophrenia. Schizophr Bull 2009;35: 336-346.

44. Tsutsumi C, Uchida H, Suzuki T, Watanabe K, Takeuchi H, Nakajima S, et al. The evolution of antipsychotic switch and polypharmacy in natural practice--a longitudinal perspective. Schizophr Res 2011;130:40-46.

45. Andreasen NC, Carpenter WT Jr, Kane JM, Lasser RA, Marder SR, Weinberger DR. Remission in schizophrenia: proposed criteria and rationale for consensus. Am I Psychiatry 2005; 162:441-449.

46. Lambert M, Karow A, Leucht S, Schimmelmann BG, Naber D. Remission in schizophrenia: validity, frequency, predictors, and patients' perspective 5 years later. Dialogues Clin Neurosci
2010;12:393-407.

47. Bobes J, Ciudad A, Alvarez E, San L, Polavieja P, Gilaberte I. Recovery from schizophrenia: results from a 1-year follow-up observational study of patients in symptomatic remission. Schizophr Res 2009; 115:58-66.

48. San L, Ciudad A, Alvarez E, Bobes J, Gilaberte I. Symptomatic remission and social/vocational functioning in outpatients with schizophrenia: prevalence and associations in a crosssectional study. Eur Psychiatry 2007;22:490-498.

49. Wunderink L, Sytema S, Nienhuis FJ, Wiersma D. Clinical recovery in first-episode psychosis. Schizophr Bull 2009;35. 362-369.

50. Lambert M, Schimmelmann BG, Naber D, Schacht A, Karow $\mathrm{A}$, Wagner $\mathrm{T}$, et al. Prediction of remission as a combination of symptomatic and functional remission and adequate subjective well-being in 2960 patients with schizophrenia. J Clin Psychiatry 2006;67:1690-1697.

51. Novick D, Haro JM, Suarez D, Lambert M, Lépine JP, Naber D. Symptomatic remission in previously untreated patients with schizophrenia: 2-year results from the SOHO study. Psychopharmacology (Berl) 2007;191:1015-1022.

52. Grech P, Taylor D. Long-term antipsychotic polypharmacy: how does it start, why does it continue? Ther Adv Psychopharmacol 2012;2:5-11.

53. Kadra G, Stewart R, Shetty H, MacCabe JH, Chang CK, Taylor $\mathrm{D}$, et al. Long-term antipsychotic polypharmacy prescribing in secondary mental health care and the risk of mortality. Acta Psychiatr Scand 2018;138:123-132.

54. Wunderink L. Personalizing antipsychotic treatment: evidence and thoughts on individualized tailoring of antipsychotic dosage in the treatment of psychotic disorders. Ther Adv Psychopharmacol 2019;9:2045125319836566.

55. Tani H, Uchida H, Suzuki T, Fujii Y, Mimura M. Interventions to reduce antipsychotic polypharmacy: a systematic review. Schizophr Res 2013;143:215-220.

56. Essock SM, Schooler NR, Stroup TS, McEvoy JP, Rojas I, Jackson C, et al. Effectiveness of switching from antipsychotic polypharmacy to monotherapy. Am I Psychiatry 2011;168: 702-708.

57. Borlido C, Remington G, Graff-Guerrero A, Arenovich T, Hazra M, Wong A, et al. Switching from 2 antipsychotics to 1 antipsychotic in schizophrenia: a randomized, double-blind, placebo-controlled study. J Clin Psychiatry 2016;77:e14-e20.

58. Constantine RJ, Andel R, McPherson M, Tandon R. The risks and benefits of switching patients with schizophrenia or schizoaffective disorder from two to one antipsychotic medication: a randomized controlled trial. Schizophr Res 2015; 166:194-200.

59. Constantine RJ, Andel R, McPherson M, Tandon R. Is the risk of antipsychotic polypharmacy discontinuation dependent on the agents used? Psychiatry Res 2018;263:238-244.

60. Tani H, Takasu S, Uchida H, Suzuki T, Mimura M, Takeuchi $\mathrm{H}$. Factors associated with successful antipsychotic dose re- 
duction in schizophrenia: a systematic review of prospective clinical trials and meta-analysis of randomized controlled trials. Neuropsychopharmacology 2020;45:887-901.

61. Correll CU, Shaikh L, Gallego JA, Nachbar J, Olshanskiy V, Kishimoto T, et al. Antipsychotic polypharmacy: a survey study of prescriber attitudes, knowledge and behavior. Schizophr Res 2011;131:58-62.

62. James BO, Omoaregba JO, Raji SO, Imishue OE, Okonoda $\mathrm{KM}, \mathrm{Nyamali} \mathrm{YI}$, et al. Attitudes towards and rationale for antipsychotic polypharmacy among psychiatrists in Nigeria: characteristics associated with high reported antipsychotic polypharmacy. Psychiatry Res 2017;248:134-139.
63. Young SL, Taylor M, Lawrie SM. "First do no harm. ” A systematic review of the prevalence and management of antipsychotic adverse effects. I Psychopharmacol 2015;29:353362.

64. Zink M, Kuwilsky A, Krumm B, Dressing H. Efficacy and tolerability of ziprasidone versus risperidone as augmentation in patients partially responsive to clozapine: a randomised controlled clinical trial. J Psychopharmacol 2009;23:305-314.

65. Pae CU. Antipsychotic polypharmacy in treatment of schizophrenia; should or should not? Chonnam Med J 2020;56:157165. 